\title{
The Effect of a Food Supplement and a Hair Lotion on the Progression of Androgenetic Alopecia
}

\author{
Michael Bayer ${ }^{1}$, Manfred Gahrtz ${ }^{2}$, Werner Voss ${ }^{1}$, Gerrit Schlippe ${ }^{1}$, Thomas Whitfield ${ }^{3}$ \\ ${ }^{1}$ Dermatest GmbH, Münster, Germany \\ ${ }^{2}$ Oxford Biolabs Deutschland GmbH, Regensburg, Germany \\ ${ }^{3}$ Oxford Biolabs Ltd., Oxford, UK \\ Email: *m.gahrtz@oxfordbiolabs.com
}

How to cite this paper: Bayer, M., Gahrtz, M., Voss, W., Schlippe, G. and Whitfield, T. (2019) The Effect of a Food Supplement and a Hair Lotion on the Progression of Androgenetic Alopecia. Journal of Cosmetics, Dermatological Sciences and Applications, 9, 292-304.

https://doi.org/10.4236/jcdsa.2019.94026

Received: October 9, 2019

Accepted: November 25, 2019

Published: November 28, 2019

Copyright () 2019 by author(s) and Scientific Research Publishing Inc. This work is licensed under the Creative Commons Attribution-NonCommercial International License (CC BY-NC 4.0). http://creativecommons.org/licenses/by-nc/4.0/ cc) (i) (8) Open Access

\begin{abstract}
Background: Progressing androgenetic alopecia (AGA), in both sexes, can result in severe distress. Treatments with the capacity to slow down the progression of AGA, or even to bring it to a halt, and at the same time don't come with side effects are consequently highly sought for. Therefore this study investigates the effect of an over-the-counter nutritional supplement and a similarly formulated topical hair lotion on the progression of AGA. Methods: Seventy-nine healthy study participants of both sexes, who were diagnosed with AGA were divided into 4 study groups. The subjects of the first group were treated with the nutritional supplement, the subjects of the second group with the topical hair lotion, the subjects of the third group with both products, and the subjects of the fourth group served as a no-treatment control. At the beginning and at the end of this nine-month study, the participants were evaluated for their hair loss status. They also answered a questionnaire for self-assessment. A part of the subjects from each study group were further analysed by phototrichography, in order to measure the number of anagen and telogen hairs. Results: It turned out that the supplement, the lotion as well as the treatment with both products not only lead to a reduction in hair loss but also to an increased anagen to telogen hair ratio, whereas no such effects could be measured for the control group. Conclusion: The results show that a systemic delivery via a nutritional supplement, as well as a follicular delivery via a topically applied lotion, both resulted in a reduced hair loss rate as well as in an increased anagen to telogen hair ratio. This demonstrates that the tested formulation is effectively slowing down the progression of AGA.
\end{abstract}




\section{Keywords}

Androgenetic Alopecia (AGA), Nutritional Supplement, Topical Hair Treatment, L-Carnitine, Zinc

\section{Introduction}

The most common form of hair loss, androgenetic alopecia (AGA), is affecting men as well as women. It mostly progresses in a patterned way, specific for the sexes: male pattern hair loss and female pattern hair loss [1]. The underlying processes are complex and only partly understood. Some of the main known factors are related to androgens. The main driver in male pattern hair loss is the fact that specific hair follicles over time become more sensitive towards dihydrotestosterone. Physiologically, this sensitivity will lead to miniaturising of the follicles, turning terminal hair into vellus hair [2]. The induced molecular processes will weaken the supply of the follicles with nutrients. In women hair loss can also specifically occur post-partum or after stopping oral contraceptive therapy [3] [4], also pointing towards a hormonal influence in hair loss. Additionally to this underlying driver, several risk factors are known. These can be age, family history, malnutrition, smoking, stress, or heavy UV radiation exposure [5] [6] [7] [8].

A head full of healthy hair signals attractiveness. People affected by hair loss thus often experience psycho-emotional stress [9]. Sufferers from hair loss, therefore, are seeking for an effective treatment, which is free from side effects. Nutrition has a profound impact on the hair condition and hair loss, as is clear from cases of hair loss caused by severe malnutrition [10]. The effects of vitamins, minerals, and physiological as well as plant secondary metabolites from nutrition or nutritional supplements on hair loss are still barely understood in detail and thus more scientific evidence is needed here [8] [11] [12].

In this study, we were testing the effect a food supplement and a similarly formulated topical hair lotion have on the progression of AGA. We did this by comparing a non-treated control group with groups that were treated either with the nutritional supplement TRX $2^{\circledast}$, with the topical TRX $2^{\circledast}$ Hair Revitalising Lotion, or with both, in subjects diagnosed with AGA, and follow the progression of hair loss in these subjects over a period of nine months. By including the topical TRX $2^{\circledast}$ lotion in the study, we were also investigating the efficacy of delivering nutrients topically via the scalp.

The main ingredients of both products are L-carnitine, zinc, niacin, biotin, and the branched-chain-amino-acids L-leucine, L-isoleucine and L-valine. Selenium is only provided by the nutritional supplement. The TRX $2^{\circledast}$ products are providing L-carnitine as L-carnitine tartrate. It is the major ingredient in both formulations. The supplement provides $800 \mathrm{mg}$ L-carnitine tartrate per day (544 $\mathrm{mg}$ L-carnitine per day), and the hair lotion is formulated with $>2 \%$ L-carnitine 
tartrate. Foitzik et al. [13] [14] reported earlier the effect L-carnitine tartrate has on hair growth. They showed the ability of L-carnitine tartrate to increase human hair growth in vitro. Increased hair shaft elongation was based on the up-regulation of the proliferation of hair matrix keratinocytes, and on the reduction of keratinocyte apoptosis. These effects lead to a prolonged anagen phase [13]. A six-month study on the in vivo application of a topical solution with $2 \%$ L-carnitine tartrate showed an increased hair density of terminal hair and an increased anagen to telogen hair ratio [14].

Zinc is another ingredient of importance that is present in both products. It has long been suspected to play a role in hair loss [12]. Serum zinc levels have been found to be reduced in subjects with AGA [15] [16]. Ozturk et al. [17] didn't find reduced serum zinc levels in male subjects with AGA, but detected significantly reduced zinc levels in hair. The exact connection between zinc and AGA is still debated, but it seems that disturbances of the zinc metabolism are associated with hair loss. Some cases show that hair loss can be successfully treated with zinc supplementation (e.g. [18]).

Similarly to zinc is the trace element selenium connected to hair loss. Selenium is required for the activity of important enzymes from the groups of glutathione peroxidases, thioredoxin reductases and iodothyronine deiodinases. These selenoenzymes take part either in the antioxidant protection of cells, in the modification of the redox status and the thyroid hormone metabolism [19]. As a lot of risk factors of hair loss, like smoking, stress or UV radiation exposure are associated to the damaging effect of reactive oxygen species (ROS) [20] [21], a compromised antioxidant-defence system, due to selenium deficiency, will likely worsen the negative effects of these risk factors.

Both products also provide niacin, a form of vitamin B3. It has been shown that a nicotinamide-rich diet leads to an increased expression of sulfonylurea receptor SUR2, an ATP-binding subunit of ATP-sensitive potassium channels [22]. A lot of evidence highlights the role such potassium channels play in AGA and hair loss in general [23] [24]. SUR2 subunits are also expressed in hair follicles [24], which might explain, why topically applied niacin derivatives improve the hair status in women with AGA [25].

Potassium channels are membrane proteins, which are composed to a high degree of hydrophobic amino acids. To these belong the three essential, branched chain amino acids L-leucine, L-isoleucine and L-valine. These three amino acids, but primarily L-leucine, stimulate protein synthesis [26]. Supplying these amino acids to the hair follicles therefore provides some of the building blocks of potassium channels and at the same time also increases general protein synthesis, thereby supporting the hair follicles' ability to increase potassium channel function.

Biotin supplementation has long time being discussed as helpful in hair growth disorders. A recent meta-analysis conducted by Patel et al. [27] confirmed the positive influence biotin has on hair and nail growth disorders. 


\section{Materials and Methods}

\section{Study subjects}

This 9-month study included 4 study groups: 1 ) the TRX $2^{\circledR}$ supplement group, 2) the $\mathrm{TRX} 2^{\circ}$ lotion group, 3) the $\mathrm{TRX} 2^{\circ}$ supplement and lotion group, and 4) the no-treatment control group. Each group was set up with 20 study subjects of both sexes. During the course of the study one subject from the control group dropped out, so that overall 79 subjects took part in the study. Table 1 gives an overview of the study participants.

The inclusion criteria were: age 18 and older, suffering from AGA, healthy skin.

The exclusion criteria were: severe or chronic skin inflammation, serious inner or chronic diseases, intake of drugs that can possibly interfere with skin reactions, application of pharmaceutical or skin care products with active ingredients until 10 days before testing, allergies or severe side effects after usage of cosmetic products, sunbath or usage of tanning bed during the study period, known cancer, pregnancy or lactation period, taking of medication with potential effect on hair growth.

The included study subjects were diagnosed with AGA and displayed a Hamilton-Norwood status [28] [29] of II to V for men and a Ludwig status of I to II for women [30].

The study was conducted in accordance with the standards of the Declaration of Helsinki and was approved by the ethics committee of the Freiburger Ethik-Kommission International (FEKI, feki Code: 016/1224). All participating study subjects were informed about the design and aim of this study, and written consent was obtained.

\section{Treatments}

The group 1-treatment with the TRX2 ${ }^{\circledR}$ supplement supplied, via three hard shell capsules, per day: $544 \mathrm{mg} \mathrm{L}$-carnitine, $150 \mathrm{mg}$ L-leucine, $100 \mathrm{mg}$ potassium, $75 \mathrm{mg}$ L-isoleucine, $75 \mathrm{mg}$ L-valine, $40 \mathrm{mg}$ nicotinamide, $15 \mathrm{mg}$ zinc, $150 \mu \mathrm{g}$ biotin, and $75 \mu \mathrm{g}$ selenium.

The group 2-treatment is the topical application of the TRX2 ${ }^{\circ}$ lotion to the af fected areas of the scalp. The TRX $2^{\circ}$ lotion is an aqueous solution, which

Table 1. Overview over study participants.

\begin{tabular}{lcccccc}
\hline \multirow{2}{*}{\multicolumn{1}{c}{ Study Group }} & \multicolumn{2}{c}{ Gender } & \multicolumn{2}{c}{ Age } & Duration of Hair Loss* \\
\cline { 2 - 7 } & Male & Female & $\begin{array}{c}\text { Mean } \\
\text { (years) }\end{array}$ & $\begin{array}{c}\text { Range } \\
\text { (years) }\end{array}$ & $\begin{array}{c}\text { Mean } \\
\text { (years) }\end{array}$ & $\begin{array}{c}\text { Range } \\
\text { (years) }\end{array}$ \\
\hline 1) TRX2 supplement & 15 & 5 & 38.2 & $21-62$ & 4.0 & $0.5-10$ \\
2) TRX2 lotion & 16 & 4 & 37.6 & $22-74$ & 4.0 & $1.0-20$ \\
3) TRX2 supplement \& lotion & 15 & 5 & 35.7 & $21-64$ & 4.8 & $0.5-15$ \\
4) No-treatment control & 14 & 5 & 42.5 & $26-67$ & 5.0 & $0.5-20$ \\
\multicolumn{1}{c}{ Sum } & 60 & 19 & 38.4 & $21-74$ & 4.4 & $0.5-20$ \\
\hline
\end{tabular}

*These data are based on self-report and are therefore prone to possibly inaccurate memory. 
provides all ingredients from the $\mathrm{TRX}^{\circ}$ supplement besides selenium: L-carnitine, L-leucine, potassium, L-isoleucine, L-valine, nicotinic acid, zinc, and biotin. It additionally provides methylsulfonylmethane (dimethyl sulfone). One $\mathrm{mL}$ of the lotion was applied to the area of thinning hair twice a day, in the morning and before bedtime.

The treatment of the subjects of group 3 , the $\mathrm{TRX}^{\circledR}$ supplement and lotion group, included the treatments of group 1 and 2 .

The subjects of group 4 stayed untreated.

\section{Study outline}

At the beginning of the study, the subjects were evaluated regarding their scalp and hair loss status by a dermatologist. Five randomly chosen subjects of each group were further analysed via phototrichography [31]. After 9 months of the respective treatment, all subjects were analysed again for their scalp and hair loss status, and the same five subjects were analysed via phototrichography again. In order to investigate the tolerability of the treatments, the study participants were additionally dermatologically evaluated after 3, 5, and 7 months. All subjects answered a questionnaire for self-assessment. Here they were asked to report adverse reactions to the supplement or the lotion and changes regarding their hair status over the period of the study. Pictures from the top view of the head were taken from all subjects at the beginning and the end of the study for a before-after comparison.

\section{Phototrichography}

The software TrichoScan ${ }^{\circledast}$ HD (DermoScan GmbH) [31] was used to evaluate hair loss. The following pre-treatment of the measuring area on the scalp was carried out:

Per subject, a measuring area was selected in the transition zone between alopecia and regularly hirsute scalp. Using a hole mask, the later measuring area was determined. The hair was threaded through the hole mask and roughly shortened with a pair of scissors. With the Moser shaver (TrichoScan ${ }^{\circledast}$ Edition), the shortened hair was shaved to an even length of $0.8 \mathrm{~mm}$. For this purpose, the razor is moved at a $90^{\circ}$ angle to the scalp without pressure.

The dyeing of the hair takes place two days after shaving: The hair dye (Goldwell Topchic $2 \mathrm{~N}$ ) was placed on a wooden spatula. The same amount of the developer (creme oxide) was added to the hair dye (1:1 mixture). Hair dye and developer were mixed well until they reach a creamy consistency. The dyeing mass is applied to the measuring area (scalp skin) of the subject and left on there to act for 15 minutes. After the contact time, the dyeing mass was removed coarsely with a swab, and the measuring area is cleaned very carefully with an alcoholic tincture (e.g. Kodan spray) and/or a soft swab.

The camera of the TrichoScan ${ }^{\circledast}$ HD is used to record the image files. The images must be free of air bubbles and surrounding hair. For the recording of the images, the measuring area is well moistened (Kodan spray). The parameters hair density $\left[1 / \mathrm{cm}^{2}\right]$, anagen hair [\%], telogen hair [\%], proportion of vellus hair [\%] and proportion of terminal hair [\%] are determined using the TrichoScan ${ }^{\circ}$ 
HD software. Per subject, one test area is measured on the scalp. Per test area, two images are evaluated by means of the TrichoScan ${ }^{\circledR} \mathrm{HD}$ analysis.

\section{Hair loss determination}

Hair loss was determined by counting lost hairs after combing. This was done as follows: After washing the hair, the still damp hair was thoroughly combed through. The hairs were collected and provided by the subject. Lost hairs were counted by the qualified study nurse. Counting took place at the indicated time points. The average per cent change in hair loss was calculated in the following way: The per cent change in hair loss from the beginning of the study to its end was determined for each study subject. Then the means of these values were calculated for each study group.

\section{Results}

\section{$\mathrm{TRX}^{\bullet}$ treatments reduce hair loss}

As food supplements may be a side-effect-less option for the treatment of AGA, we investigated the effects of an L-carnitine based food supplement and a similarly formulated topical hair lotion on the progression of AGA in comparison to an untreated control.

When comparing the hair loss status of the four study groups after 9-month, to the baseline, it turned out that on average all three groups that received a treatment, had a reduced hair loss rate, but not the no-treatment control. As is depicted in Figure 1 the average per cent hair loss reduction was in group 1 (supplement) $-35.4 \%$, in group 2 (lotion) $-38.8 \%$, and in group 3 (supplement and lotion) $-31.0 \%$, whereas in group 4 (no-treatment control) the average per cent hair loss had increased by $+23.7 \%$.

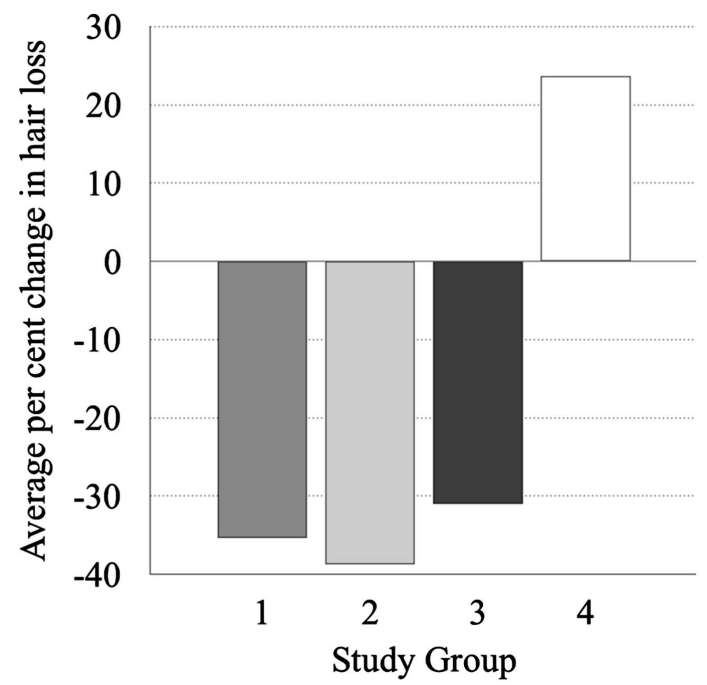

Figure 1. Average per cent change in hair loss. The average per cent change in hair loss after 9 months, compared to the baseline, for each of the four study groups is shown here: $\square$ : Study Group $1-\mathrm{TRX}^{\circledast}$ supplement: $-35.4 \%$; $\square$ : Study Group 2-TRX2 ${ }^{\circledR}$ lotion: $-38.8 \%$; $\square$ : Study Group 3-TRX2 ${ }^{\circledast}$ supplement and lotion: $-31.0 \%$; $\square$ : Study Group 4-no-treatment control: $+23.7 \%$. 
In accordance with the hair loss determination is the fact that $75 \%$ of the study subjects from group 1 to 3 reported a reduced hair loss at the end of the study compared to its beginning. Also, $67 \%$ of them reported that they perceived their hair healthier at the end of the study.

\section{$\mathrm{TRX}^{\circ}$ treatments increase the anagen to telogen hair ratio}

The higher the hair loss rate is, the lower is the ratio of anagen to telogen hairs (A/T ratio). Because in the treatment groups 1 to 3 a reduced hair loss was recorded, a higher A/T ratio should be expected. Indeed, that is exactly what the phototrichography found out. As is shown in Figure 2, the average per cent change in the anagen to telogen hair ratio was in group 1 (supplement) $+55.1 \%$, in group 2 (lotion) $+39.4 \%$, and in group 3 (supplement and lotion) $+63.5 \%$, whereas in group 4 (no-treatment control) the average per cent change in the $\mathrm{A} / \mathrm{T}$ ratio decreased by $-3.9 \%$.

\section{Exemplary cases}

Figures 3-6 show each one exemplary case from each of the four study groups, respectively. Given are the before and after pictures of the head top-view and the TrichoScan ${ }^{\oplus}$ images as well as their analysis. These examples demonstrate and underline the above-shown effects on the progression of AGA in the four study groups.

\section{Skin tolerance and compatibility}

During the course of the study all of the 60 study participants who received treatment with the supplement and/or the hair lotion were found to tolerate the products very well under dermatological and clinical criteria. There were no undesired or even pathological skin reactions in the test area.

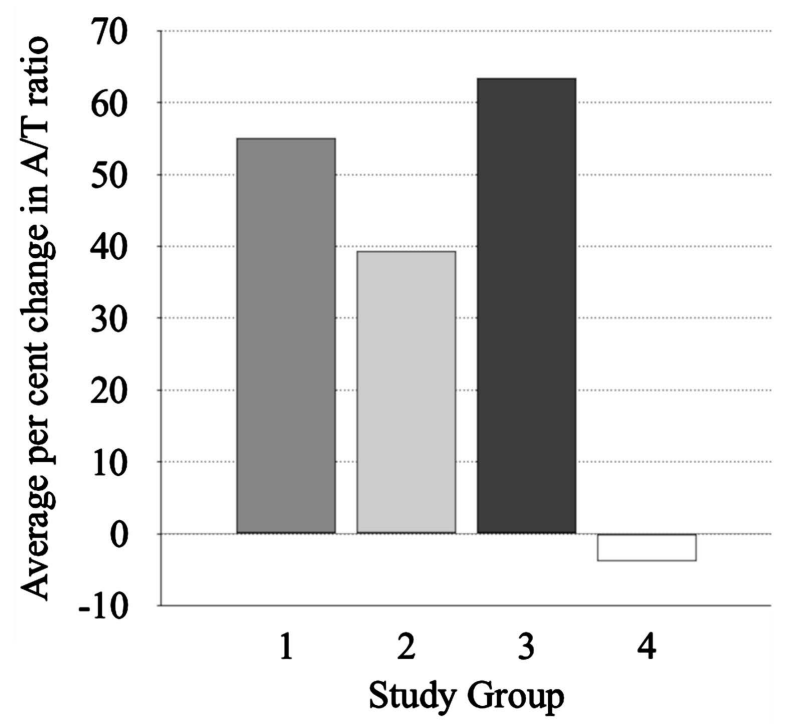

Figure 2. Average per cent change in A/T ratio. The average per cent change in the anagen to telogen hair ratio after 9 months, compared to the baseline, for each of the four study groups is shown here: $\square$ : Study Group $1-\mathrm{TRX}^{\varpi}$ supplement: $+55.1 \%$; $\square$ : Study Group 2-TRX2 ${ }^{\oplus}$ lotion: $+39.4 \%$; $\square$ : Study Group 3-TRX2 $2^{\oplus}$ supplement and lotion: +63.5\%; $\square$ : Study Group 4-no-treatment control: $-3.9 \%$. 


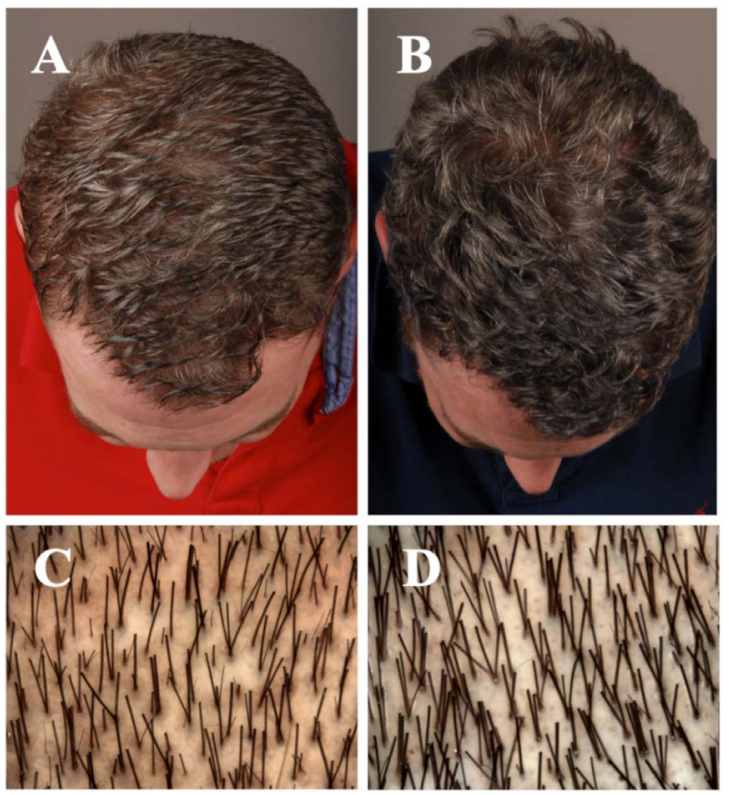

Figure 3. Exemplary case from study group 1 (supplement). Male study subject, 37 years old, diagnosed with AGA, Norwood I. A and B: Before/after pictures: A: Taken at the beginning of the study; B: taken at the end of the study. C and D: TrichoScan ${ }^{\circledR}$ images: C: baseline at the beginning of the study; D: after 9 month of treatment with TRX2 ${ }^{\oplus}$ food supplement. Whereas the total number of hairs was reduced by $-1.9 \%$, and the number of vellus hairs decreased by $-44.9 \%$, the numbers of terminal hair increased by $+12.4 \%$. During the study period the anagen hair rate increased from $75.1 \%$ to $86.1 \%$.

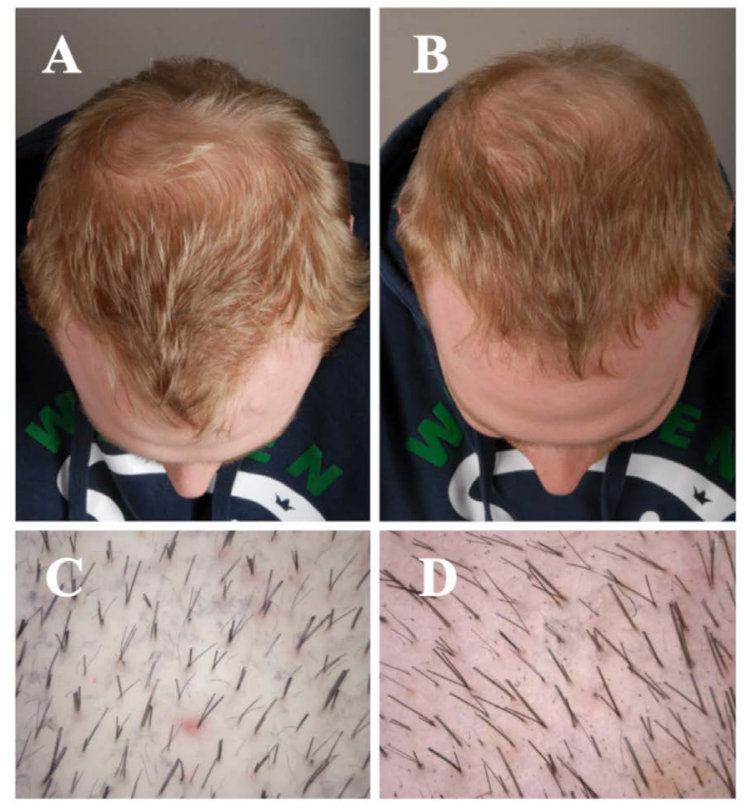

Figure 4. Exemplary case from study group 2 (hair lotion). Male study subject, 26 years old, diagnosed with AGA, Norwood IV. A and B: Before/after pictures: A: Taken at the beginning of the study; B: taken at the end of the study. C and D: TrichoScan ${ }^{\circledast}$ images: C: baseline at the beginning of the study; D: after 9 month of treatment with TRX2 ${ }^{\circledR}$ Hair Revitalising Lotion. The total number of hairs increased by $+12.7 \%$, the number of vellus hairs increased by $+350 \%$, and the numbers of terminal hair increased by $+9.1 \%$. During the study period the anagen hair rate increased from $31.0 \%$ to $58.0 \%$. 

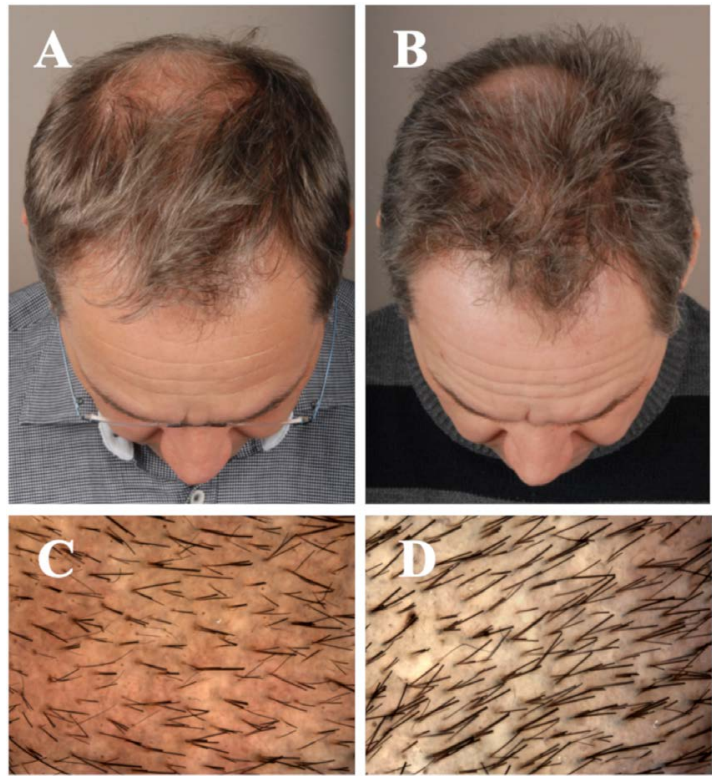

Figure 5. Exemplary case from study group 3 (supplement and hair lotion). Male study subject, 46 years old, diagnosed with AGA, Norwood V. A and B: Before/after pictures: A: Taken at the beginning of the study; B: taken at the end of the study. C and D: TrichoS$\mathrm{can}^{\otimes}$ images: C: baseline at the beginning of the study; D: after 9 month of treatment with TRX $2^{\circledast}$ supplement and Hair Revitalising Lotion. The total number of hairs increased by $+31.6 \%$, the number of vellus hairs decreased by $-32.3 \%$, and the numbers of terminal hair increased by $+117.3 \%$. During the study period the anagen hair rate increased from $38.8 \%$ to $64.4 \%$.
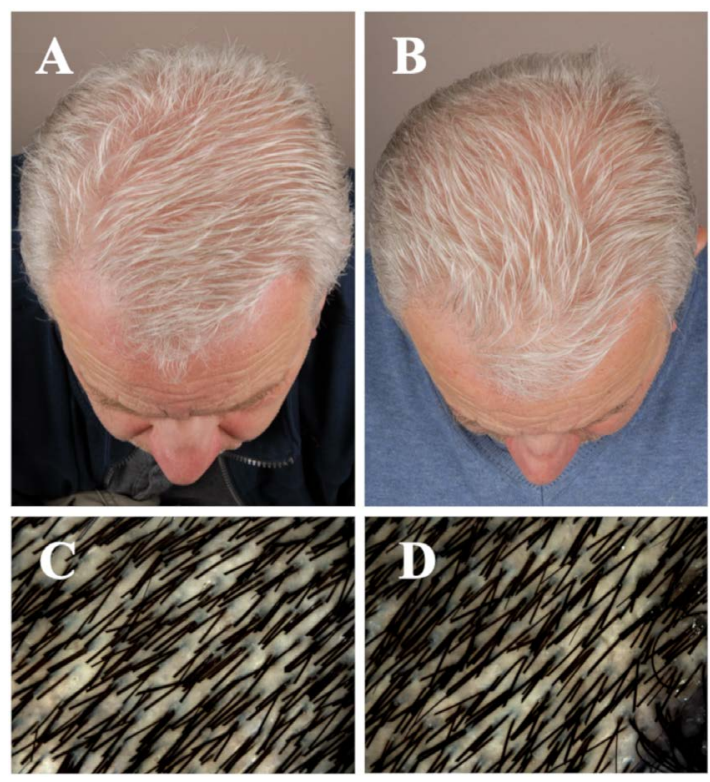

Figure 6. Exemplary case from study group 4 (no-treatment control). Male study subject, 59 years old, diagnosed with AGA, Norwood V - VI. A and B: Before/after pictures: A: Taken at the beginning of the study; B: taken at the end of the study. C and D: TrichoScan ${ }^{\circledast}$ images: C: baseline at the beginning of the study; D: after 9 month. The total number of hairs decreased by $-24.1 \%$, the number of vellus hairs decreased by $-24.4 \%$, and the numbers of terminal hair decreased by $-30.0 \%$. During the study period the anagen hair rate decreased from $76.9 \%$ to $75.2 \%$. 


\section{Discussion}

In order to test the ability of a nutritional supplement and a similarly formulated topical lotion to positively influence the progression of AGA, we tested the $\mathrm{TRX}^{\circ}$ nutritional supplement and the $\mathrm{TRX}^{\oplus}$ Hair Revitalising Lotion, over a period of nine months, in a setting with four study groups, from which one served as a no-treatment control. It turned out that the supplement, the lotion, as well as the treatment with both products, not only lead to a reduction in hair loss but also to an increased anagen to telogen hair ratio, whereas no such effects could be measured for the control group.

Our results reported here (Figure 1 and Figure 2), especially from the usage of the TRX2 ${ }^{\circ}$ Hair Revitalising Lotion, are in line with the findings from Foitzik et al. [13] [14] on the in vitro as well as in vivo effect of L-carnitine tartrate on hair growth. As both products additionally provide zinc, niacin, biotin, and the branched chain amino acids L-leucine, L-isoleucine and L-valine, which are also connected to a good hair status, the measured effects are likely dependent on additive or even synergistic effects of the mentioned ingredients.

The similar effects resulting from the treatments with the supplements and with the lotion clearly indicate that the topical application successfully delivers the active ingredients to the hair follicle to a similar degree as the systemic application. Whereas it is unlikely that the topical preparation as such immediately and directly reaches the hair bulb region, it has been shown that drug delivery to the skin occurs also through the hair follicles [32]. Thereby the sebaceous gland serves as a long-term reservoir [33]. In the case of topically applied caffeine, its delivery could be shown after only 5 minutes, but only with open hair follicles. In the case, these had been blocked, significant amounts of caffeine needed about 30 minutes to be detected [34]. If the provided concentrations of the actives are high enough, and the topical product is repeatedly applied as a leave-on product, like in the current study, we believe that sufficient amounts of actives reach the hair bulb. Generally, it can be stated that follicular targeting of actives is becoming a promising tool in dermatotherapy [35].

The obtained data do not allow evaluating, whether the TRX2 ${ }^{\circ}$ treatments work with different efficacy in men and women, as the number of participating women was too low.

Due to the emotional stress, which AGA creates, effective solutions that are free of side effects are in high demand. This study shows that a systemic delivery via a nutritional supplement, as well as a follicular delivery via topical application, of a combination of L-carnitine tartrate, zinc, niacin, branched chain amino acids and biotin, resulted in a reduced hair loss rate as well as in an increased anagen to telogen hair ratio. This proves that the tested formulation is effectively slowing down the progression of AGA. This immediately leads to the conclusion that such treatments are most effective when used from the early stages of AGA on. Further studies can help to elucidate the age, gender or speed of progression of hair loss, which is most responsive to the $\mathrm{TRX} 2^{\circledR}$ treatment. 


\section{Acknowledgements}

We thank Dr. Lydia Braun, University of Regensburg, for helpful comments on the manuscript.

\section{Conflicts of Interest}

MG and TW work in the company, which commercializes both products tested in the present study.

\section{References}

[1] Gupta, M. and Mysore, V. (2016) Classification of Patterned Hair Loss: A Review. Journal of Cutaneous and Aesthetic Surgery, 9, 3-12. https://doi.org/10.4103/0974-2077.178536

[2] Paus, R. and Cotsarelius, G. (1999) The Biology of Hair Follicles. The New England Journal of Medicine, 341, 491-497. https://doi.org/10.1056/NEJM199908123410706

[3] Lynfield, Y.L. (1960) Effect of Pregnancy on the Human Hair Cycle. Journal of Investigative Dermatology, 35, 323-327. https://doi.org/10.1038/jid.1960.127

[4] Dawber, R.P.R. and Connor, B.L. (1971) Pregnancy, Hair Loss and the Pill. British Medical Journal, 4, 234. https://doi.org/10.1136/bmj.4.5781.234

[5] Hadshiew, I.M., Foitzik, K., Arck, P.C. and Paus, R. (2004) Burden of Hair Loss: Stress and the Underestimated Psychosocial Impact of Telogen Effluvium and Androgenetic Alopecia. Journal of Investigative Dermatology, 123, 455-457. https://doi.org/10.1111/j.0022-202X.2004.23237.x

[6] Rajput, R.J. (2017) Role of Non Androgenic Factors in Hair Loss and Hair Regrowth. Journal of Cosmetology \& Trichology, 3, 1. https://doi.org/10.4172/2471-9323.1000118

[7] Su, L.-H. and Chen, T.H.-H. (2007) Association of Androgenetic Alopecia with Smoking and Its Prevalence among Asian Men. A Community-Based Survey. Archives of Dermatology, 143, 1401-1406. https://doi.org/10.1001/archderm.143.11.1401

[8] Trüeb, R.M. (2015) Effect of Ultraviolet Radiation, Smoking and Nutrition on Hair. Current Problems in Dermatology, 47, 107-120. https://doi.org/10.1159/000369411

[9] Danyal, M., Shah, S.I.A., Hassan, M.S.U. and Qureshi, W. (2018) Impact of Androgenetic Alopecia on the Psychological Health of Young Men. Pakistan Journal of Medical and Health Sciences, 12, 406-410.

[10] Finner, A.M. (2013) Nutrition and Hair, Deficiencies and Supplements. Dermatologic Clinics, 31, 167-172. https://doi.org/10.1016/j.det.2012.08.015

[11] Almohanna, H.M., Ahmed, A.A., Tsatalis, J.P. and Tosti, A. (2018) The Role of Vitamins and Minerals in Hair Loss: A Review. Dermatology and Therapy, 9, 51-70. https://doi.org/10.1007/s13555-018-0278-6

[12] Goldberg, L.J. and Lenzy, Y.L. (2010) Nutrition and Hair. Clinics in Dermatology, 28, 412-419. https://doi.org/10.1016/j.clindermatol.2010.03.038

[13] Foitzik, K., Hoting, E., Förster, T., Pertile, P. and Paus R. (2007) L-Carnitine-L-Tartrate Promotes Human Hair Growth in Vitro. Experimental Dermatology, 16, 936-945. https://doi.org/10.1111/j.1600-0625.2007.00611.x

[14] Foitzik, K., Hoting, E. Heinrich, U., Tronnier, H. and Paus, R. (2007) Indications that Topical L-Carnitin-L-Tartrate Promotes Human Hair Growth in Vivo. Journal 
of Dermatological Science, 48, 141-144.

https://doi.org/10.1016/j.jdermsci.2007.07.001

[15] El-Esawy, F.M., Hussein, M.S. and Mansour, A.I. (2019) Serum Biotin and Zinc in Male Androgenetic Alopecia. Journal of Cosmetic Dermatology, 18, 1546-1549. https://doi.org/10.1111/jocd.12865

[16] Kil, M.S., Kim, C.W. and Kim, S.S. (2013) Analysis of Serum Zinc and Copper Concentrations in Hair Loss. Annals of Dermatology, 25, 405-409. https://doi.org/10.5021/ad.2013.25.4.405

[17] Ozturk, P., Kurutas, E., Ataseven, A., Dokur, N., Gumusalan, Y., Gorur, A., Tamer, L. and Inaloz, S. (2014) BMI and Levels of Zinc, Copper in Hair, Serum and Urine of Turkish Male Patients with Androgenetic Alopecia. Journal of Trace Elements in Medicine and Biology, 28, 266-270. https://doi.org/10.1016/j.jtemb.2014.03.003

[18] Alhaj, E., Alhaj, N. and Alhaj, N.E. (2007) Diffuse Alopecia in a Child due to Dietary Zinc Deficiency. SKINmed: Dermatology for the Clinician, 6, 199-200. https://doi.org/10.1111/j.1540-9740.2007.05881.x

[19] Beckett, G.J. and Arthur, J.R. (2005) Selenium and Endocrine Systems. Journal of Endocrinology, 184, 455-465. https://doi.org/10.1677/joe.1.05971

[20] Prie, B.E., Iosif, L., Tivig, I., Stoian, I. and Giurcaneanu, C. (2016) Oxidative Stress in Androgenetic Alopecia. Journal of Medicine and Life, 9, 79-83.

[21] Upton, J.H., Hannen, R.F., Bahta, A.W., Farjo, N., Farjo, B. and Philpott, M.P. (2015) Oxidative Stress-Associated Senescence in Dermal Papilla Cells of Men with Androgenetic Alopecia. Journal of Investigative Dermatology, 135, 1244-1252. https://doi.org/10.1038/jid.2015.28

[22] Sukhodub, A., Sudhir, R., Du, Q., Jovanović, S., Reyes, S. and Jovanović, A. (2011) Nicotinamide-Rich Diet Improves Physical Endurance by up-Regulating SUR2A in the Heart. Journal of Cellular and Molecular Medicine, 15, 1703-1712. https://doi.org/10.1111/j.1582-4934.2010.01156.x

[23] Buhl, A.E., Waldon, D.J., Conrad, S.J., Mulholland, M.J., Shull, K.L., Kubicek, M.F., Johnson, G.A., Brunden, M.N., Stefanski, K.J., Stehle, R.G., Gadwood, R.C., Kamdar, B.V., Thomasco, L.M., Schostarez, H.J., Schwartz, T.M. and Diani, A.R. (1992) Potassium Channel Conductance: A Mechanism Affecting Hair Growth Both in Vitro and in Vivo. Journal of Investigative Dermatology, 98, 315-319.

[24] Shorter, K., Farjo, N.P., Picksley, S.M. and Randall, V.A. (2008) Human Hair Follicles Contain Two Forms of ATP-Sensitive Potassium Channels, Only One of Which Is Sensitive to Minoxidil. FASEB Journal, 22, 1725-1736. https://doi.org/10.1096/fj.07-099424

[25] Draelos, Z.D., Jacobson, E.L., Kim, H., Kim, M., Jacobson, M.K. (2005) A Pilot Study Evaluating the Efficacy of Topically Applied Niacin Derivatives for Treatment of Female Pattern Alopecia. Journal of Cosmetic Dermatology, 4, 258-261. https://doi.org/10.1111/j.1473-2165.2005.00201.x

[26] Kimball, S.R. and Jefferson, L.S. (2006) Signaling Pathways and Molecular Mechanisms through Which Branched-Chain Amino Acids Mediate Translational Control of Protein Synthesis. The Journal of Nutrition, 136, 227S-231S. https://doi.org/10.1093/jn/136.1.227S

[27] Patel, D.P., Swink, S.M. and Castelo-Soccio, L. (2017) A Review of the Use of Biotin for Hair Loss. Skin Appendage Disorders Guidelines, 3, 166-169. https://doi.org/10.1159/000462981

[28] Hamilton, J.B. (1951) Patterned Loss of Hair in Man: Types and Incidence. Annals of the New York Academy of Sciences, 53, 708-728. 
https://doi.org/10.1111/j.1749-6632.1951.tb31971.x

[29] Norwood, O.T. (1975) Male Pattern Baldness: Classification and Incidence. Southern Medical Journal, 68, 1359-1365. https://doi.org/10.1097/00007611-197511000-00009

[30] Ludwig, E. (1977) Classification of the Types of Androgenetic Alopecia (Common Baldness) Occurring in the Female Sex. British Journal of Dermatology, 97, 247-254. https://doi.org/10.1111/j.1365-2133.1977.tb15179.x

[31] Hoffmann, R. (2003) TrichoScan: A Novel Tool for the Analysis of Hair Growth in Vivo. Journal of Investigative Dermatology Symposium Proceedings, 8, 109-115. https://doi.org/10.1046/j.1523-1747.2003.12183.x

[32] Wosicka, H. and Cal, K. (2010) Targeting to the Hair Follicles: Current Status and Potential. Journal of Dermatological Science, 57, 83-89. https://doi.org/10.1016/j.jdermsci.2009.12.005

[33] Lademann, J., Richter, H., Schaefer, U.F., Blume-Peytavi, U., Teichmann, A., Otberg, A. and Sterry, W. (2006) Hair Follicles-A Long-Term Reservoir for Drug Delivery. Skin Pharmacology and Physiology, 19, 232-236.

https://doi.org/10.1159/000093119

[34] Otberg, N., Teichmann, A., Rasuljev, U., Sinkgraven, R., Sterry, W. and Lademann, J. (2007) Follicular Penetration of Topically Applied Caffeine via a Shampoo Formulation. Skin Pharmacology and Physiology, 20, 195-198.

https://doi.org/10.1159/000101389

[35] Vogt, A., Mandt, N., Lademann, J., Schaefer, H. and Blume-Peytavi, U. (2005) Follicular Targeting-A Promising Tool in Selective Dermatotherapy. Journal of Investigative Dermatology Symposium Proceedings, 10, 252-255.

https://doi.org/10.1111/j.1087-0024.2005.10124.x 